\title{
Perception of the head transversal plane and the subjective horizontal during gondola centrifugation
}

\author{
ARNE TRIBUKAIT \\ Karolinska Hospital, Stockholm, Sweden \\ and Defense Research Agency, Stockholm, Sweden \\ and \\ OLA EIKEN \\ Defense Research Agency, Stockholm, Sweden
}

\begin{abstract}
The subjective visual horizontal (SVH) and the subjective head transversal plane (STP) were measured by means of an adjustable luminous line in darkness during centrifuging. Subjects $(N=10)$ were seated upright, facing forward in a swing-out gondola. After acceleration of the centrifuge to $2 \mathrm{G}$ (vectorial sum of the earth's gravity and the centrifugal force; gondola inclination $60^{\circ}$ ), subjects had to set the line either so that it was perceived as gravitoinertially horizontal (SVH) or so that it was perceived as parallel with the transversal ("horizontal") plane of the head (STP). Initially after acceleration, the SVH was tilted with respect to the gravitoinertial horizontal of the gondola $\left(M=16.6^{\circ}\right)$. This tilt was compensatory with respect to the gondola inclination. However, the STP was tilted in the opposite direction $\left(M=12.4^{\circ}\right)$, which might suggest a vestibular-induced distortion of the mental representation of one's own body. Similar results were obtained when measuring the subjective visual vertical (SVV) and the subjective midsagittal plane (SSP) in 5 subjects. The perceived roll angle (obtained as SVH-STP or SVV-SSP) was considerably larger than had previously been reported. Time constants for exponential decay of the tilt of the SVH or SVV were often 2-3 min, indicating a memory for semicircular canal information on changes in head orientation-a position-storage mechanism.
\end{abstract}

Spatial orientation denotes the ability to judge the position and movement of an object relative to a reference system. The object may be one's own body, a part of the body, or an external object. Reference systems of special importance are (1) the coordinate system provided by the surface of the earth and the direction of gravity and (2) a coordinate system fixed with respect to one's own body and consisting of the three main body axes. If an individual, when judging the position of a visual object, relates to the former, his or her way of orienting may be termed allocentric (Holdstock et al., 2000). In contrast, relating to the coordinate system of one's own body implies an egocentric way of orienting (Howard \& Templeton, 1966; Vallar et al., 1999).

Spatial orientation in humans is often studied psychophysically by means of visual indicators. This way of testing is based on the assumption that the subject has a mental representation of some physically definable entity, and that he or she is able to report this mental repre-

This study was supported by the Swedish Defense Material Administration. We thank Michael Crisp, Bertil Lindborg, and Björn Johannesson for technical assistance. Correspondence concerning this article should be addressed to A. Tribukait, Department of Audiology, Karolinska Hospital, SE 17176 Stockhom, Sweden (e-mail: arne.tribukait (a)foi.se). sentation by adjusting a visual indicator. Two things of particular interest are the direction of gravity and the length axis of one's own body. Regarding the former, the subject has to adjust a luminous line, at a straight-ahead eye-level position and rotable about the visual axis, so that it is perceived as vertical or horizontal. The deviation, measured in degrees, from veridicality is termed the subjective visual vertical (SVV) or subjective visual horizontal (SVH). Thus, by this way of testing it is possible to obtain quantitative data on certain mental states; adjustments of the line are a commonly accepted measure of the mental representation of the direction of gravity.

In normal subjects, seated upright in darkness, the SVH or SVV does not deviate more than $2.5^{\circ}$ from the gravitational horizontal or vertical (Böhmer \& Rickenmann, 1995; Dai, Curthoys, \& Halmagyi, 1989; Tribukait, Bergenius, \& Brantberg, 1998). This measure of spatial orientation depends mainly on the vestibular organ of the inner ear (H. Mittelstaedt, 1991; H. Mittelstaedt \& Fricke, 1988; Schöne, 1964). A tilt of the SVH or SVV in a normalsighted subject, who is seated upright, suggests an asymmetry in the resting neural activity of the vestibular system. This may, for instance, be caused by a unilateral loss of peripheral vestibular function (Dai et al., 1989) or by central nervous lesions (Brandt \& Dieterich, 1987). Furthermore, the magnitude of tilt of SVH is likely to cor- 
respond to the degree of asymmetry in resting neural activity. It is therefore possible to study the time course of recovery after a unilateral vestibular lesion, which is reflected in a diminution in the tilt of SVH during weeks or months (Dai et al., 1989; Tribukait et al., 1998).

Thanks to a compensation function, using information from the otolith receptors, at moderate static lateral head tilts $\left(<30^{\circ}\right)$ subjects may still set the luminous line approximately horizontally or vertically (Tribukait, Bergenius, \& Brantberg, 1996; Udo de Haes, 1970). At larger tilts $\left(60^{\circ}-90^{\circ}\right)$, however, there is often an underestimation of the head and body tilt so that the subjective horizontal or vertical deviates by $20^{\circ}-30^{\circ}$ from veridicality (Mittelstaedt, 1991; Udo de Haes, 1970). This means that the otolith system does not correctly estimate the magnitude of head tilt.

In spite of such errors in an allocentric orientation task, subjects may still correctly indicate, by adjusting a luminous line, the orientation of the body length axis (Haustein, 1992), which is an egocentric orientation task. This implies that the visual system gives a correct representation of the line relative to the head also in head positions for which the nervous system does not correctly estimate the direction of gravity (H. Mittelstaedt, 1983). It is of particular interest that this ability to indicate the orientation of one's own body may be adequate even when the perception of one's orientation with respect to gravitational vertical is erroneous. It suggests a certain integrity of such a mental entity as the representation of the spatial extension of one's own body.

However, it has been reported that the mental representation of the midsagittal plane may be influenced by semicircular canal stimulation (Bottini et al., 2001; Rorden, Karnath, \& Driver, 2001). Patients with lesions in the parietal lobe often have a reduced ability to direct attention to the side of space opposite the lesion. It has been proposed that this so-called hemineglect consists in a pathological rotation of egocentric space (Karnath, 1997). Semicircular canal stimulation by means of coldwater irrigation of the contralesional ear reduces the degree of neglect, which is reflected, for instance, in a change in the subjective straight-ahead in pointing tasks. Such distortions in the mental representation of the main planes, or axes, of one's own body, revealed in egocentric orientation tasks, could theoretically have an indirect effect on the results of allocentric tasks, such as indicating the SVH or SVV, as well.

When one is performing a change in head position with respect to gravity, there is usually a redundancy of information from the semicircular canals and the otolith

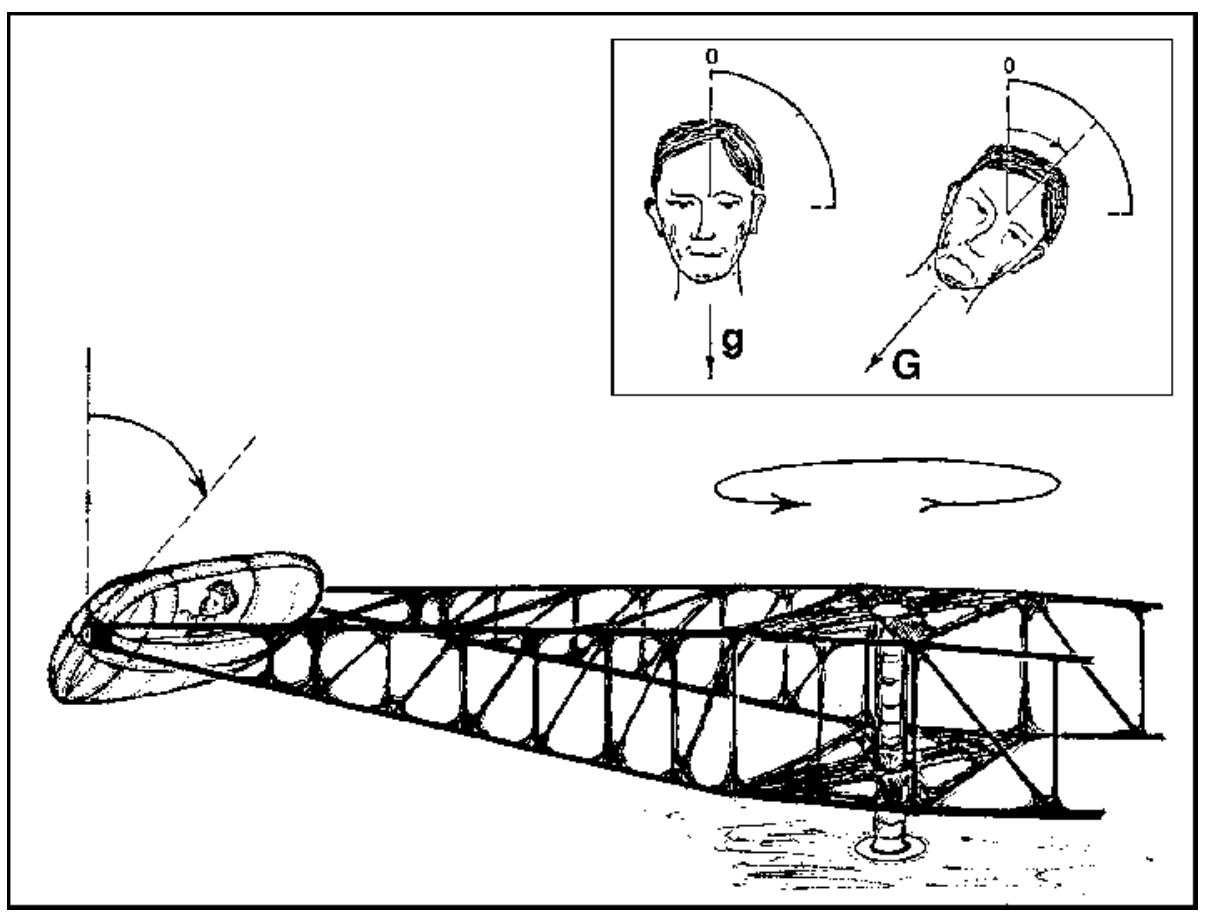

Figure 1. The centrifuge. Rotation of the centrifuge is counterclockwise, as seen from above, with the subject facing forward. During acceleration, the cabin is rolled so that the resultant gravitoinertial force vector (vector sum of the earth's gravity and the centrifugal force) remains aligned with the subject's head-to-seat $(z)$ axis. Therefore, the otolith system does not receive a roll-tilt stimulus. However, the change in roll position (the inclination) of the cabin and subject with respect to the centrifuge main axis is a roll-plane semicircular-canal stimulus. Hence, acceleration of the centrifuge creates a conflict between the otolith organs, sustainedly signaling upright position, and the semicircular canals, signaling a change in $\mathrm{roll} / \mathrm{head}$ position to the left. 
organs (Stockwell \& Guedry, 1970). The semicircular canals signal for the angular change in position, the otolith organs sense the head position prior to and after the movement. This cooperation between the two separate receptor systems is probably important for an instantaneous, accurate, and lasting sensation of changes in head position.

However, in certain conditions, such as in moving vehicles, the interplay between the otolith organs and semicircular canals may be disturbed. For instance, in weightlessness angular changes in head position will be adequately sensed by the semicircular canals but not by the otolith organs. Also, when a pilot is entering a coordinated bank and turn, the vertical semicircular canals sense the change in roll (frontal plane) position, whereas the otolith organs continually signal for upright position. A similar stimulus situation may occur in a large human centrifuge. If a subject is seated upright and facing forward in a swing-out gondola (Figure 1), the length axis of the head and body will continually remain parallel with the gravitoinertial force (vectorial sum of the earth's gravity and the centrifugal force). Therefore, during acceleration of the centrifuge the otolith organs will not sense a lateral head tilt. However, the swing-out of the gondola during acceleration of the centrifuge is a rollplane stimulus to the semicircular canals, such as when the head is tilted laterally (Glasauer, 1993; Guedry \& Oman, 1990; McGrath, Guedry, Oman, \& Rupert, 1995). This causes a conflict between the otolith organs and the semicircular canals. During acceleration of the centrifuge, there is, in addition, a complex angular-velocity stimulation of the semicircular canal system; initially, this consists mainly in yaw- (horizontal) plane rotation, but, due to the inclination of the gondola, at $2 \mathrm{G}$ the pitch(sagittal-) plane component dominates. The perceptual consequences of such intravestibular conflicts are of interest not only physiologically but also regarding the ability to maintain orientation when flying in aircraft or space shuttles.

If the direction of rotation of the centrifuge is counterclockwise, as seen from above, the gondola and subject will roll to the left during acceleration of the centrifuge. At $2 \mathrm{G}$, the inclination of the gondola is $60^{\circ}$. If the subject were able to maintain a correct imagination of the external world, he or she would set the luminous line tilted by $60^{\circ}$ to the right with respect to the gravitoinertial horizontal inside the gondola. A gradual "forgetting" of the initial change in roll position would be reflected in a diminution in the tilt of SVH. If the subject had no sensation of being rolled, the SVH would coincide with the gravitoinertial horizontal.

In an earlier study (Tribukait, 1999), it was found that acceleration in a gondola centrifuge caused a tilt of the SVH, which was compensatory with respect to the inclination of the gondola. The magnitude of tilt was small in comparison with the gondola inclination, and it decayed exponentially, approaching the gravitoinertial horizontal within a few minutes. The subjects, being naive about gondola centrifuging, had been instructed to indicate what they spontaneously perceived as the horizontal of the external world. However, some of them reported that they were slightly confused or startled during acceleration of the centrifuge and that they therefore tried to adjust the line to coincide with their own head-transversal plane. This could possibly represent a more primitive attitude elicited in a disorienting or distressing situation. Conspicuously, in such cases the settings of the line did not coincide with the true orientation of the head. Rather, the STP was tilted in the direction opposite that of the SVH. This implies that the semicircular canal stimulation during acceleration had induced a distortion of the mental representation of one's own body, which contrasts with the above-mentioned results during static lateral tilt in $1 \mathrm{~g}$ environment. The data were, however, too scant to permit any comparison between the SVH and the STP regarding the magnitude of initial tilt and the time constant for exponential decay.

The present investigation aimed at a further characterization of such effects of acceleration in a gondola centrifuge. Specifically, we wanted to (1) establish whether there is a consistent relationship between the instructions given to the subject and the settings of the luminous line, (2) compare the SVH and STP with respect to magnitude of the initial tilt and time constant for exponential decay, (3) calculate the perceived change in roll position as the difference between the SVH and STP, and (4) establish whether measuring the SVV and the perceived orientation of the SSP would give corresponding results.

\section{METHOD}

\section{Subjects}

Thirteen healthy subjects, 6 females and 7 males, 20-37 years of age, were recruited for the study. Ten of the subjects participated in a first series of experiments on the SVH and STP. In a second series, the SVV and SSP were measured in 5 of the subjects. The subjects did not have any history of vertigo, hearing loss, or neurological disturbances and were free of medications. They did not have any experience of maneuvering an aircraft. The subjects gave their informed consent and were free to withdraw at any time during the experiment. The test procedure was in accordance with the Declaration of Helsinki and was approved by the human ethics committee at the Karolinska Institute.

\section{Centrifugation}

The experiments were performed in the human centrifuge at the Karolinska Institute, Stockholm. The radius of the centrifuge is $7.25 \mathrm{~m}$. A swing-out gondola is fixed on a pivot, whose axis is tangential so that the gondola is deflected outward in the direction of the resultant force vector (resultant of earth's gravity and the centrifugal force). The subjects were fixed in a cockpit seat by means of safety belts. The head position was fixed by use of a head holder, which was adjusted so that a line from the external auditory meatus to the inferior margin of the orbit was tilted upward (nose up) $10^{\circ}$ with respect to the gravitational horizontal. The interocular line was gravitationally horizontal. No bite bar was used due to the necessity of being able to communicate with the subjects during the runs and in order to minimize discomfort; in case of anxiety or motion sickness, a bite bar can increase the discomfort.

The rotation of the centrifuge is counterclockwise (as seen from above) with the subject facing forward (see Figure 1). During cen- 
trifuging, the vertical axis of the gondola, and hence the long axis $(z$-axis) of the subject, is always aligned with the resultant force vector of the earth's gravity and the centrifugal force. Continuous monitoring showed that the vertical axis of the gondola did not deviate by more than $1.0^{\circ}$ from the gravitoinertial vertical and that the position was perfectly stable; that is, it did not change by more than $0.1^{\circ}$ during the entire $2 \mathrm{G}$ plateau. Early in the acceleration phase, there was a transient deviation $\left(2^{\circ}-3^{\circ}\right)$ from the ideal; due to a frictionbased damping mechanism (aimed at preventing the gondola from oscillating after rapid accelerations), the gondola tended to lag slightly behind the $\mathrm{G}$ vector when this was still close to $1.0 \mathrm{~g}$. The pivot did not permit any change in pitch position of the gondola.

With an angular acceleration of $15 \% \mathrm{sec}^{2}$ the settled gravitoinertial force level of $2 \mathrm{G}$ was attained in $5.85 \mathrm{sec}$. The change in roll position was $60^{\circ}$ and during acceleration of the centrifuge, the roll angular speed was approximately $10 \%$ sec. Figure 2 shows changes with time of the angular velocity of the centrifuge about its main axis, the gondola inclination, the magnitude of the resultant gravitoinertial force vector, the angular velocity component in the subject's yaw plane (angular velocity of the centrifuge $\times$ the cosine of the gondola inclination), and the angular velocity component in the subject's pitch plane (angular velocity of the centrifuge $\times$ the sine of the gondola inclination).

The recording equipment was located in the experimenter's room, separated from the centrifuge well. The subject was observed in infrared light by means of a video camera, and he or she always had the possibility to communicate with the experimenter by means of a two-way intercom system. The subject's heart rate and rhythm were monitored continuously by means of electrocardiography.

\section{Measurements of the SVH, STP, SVV, and SSP}

In front of the subject (at a distance of $0.9 \mathrm{~m}$ from his or her eyes), a narrow luminous line of red light-emitting diodes subtended a visual angle of $6.5^{\circ}$. The line could be rotated, by the experimenter as well as by the subject, about the naso-occipital (visual) axis. The subject was asked to adjust the line by means of two push buttons on a remote control every time it was switched on. When pleased with a setting, the subject pressed a third button, which extinguished the line. The deviation of the line from the gravitoinertial horizontal or vertical was then recorded with an accuracy of $0.1^{\circ}$. Before the line was switched on again, it was rotated $5^{\circ}-20^{\circ}$ (randomly) alternatively to the right and to the left. The subjects were able to make four settings of the line per minute. Except for the line, the gondola was completely darkened.

\section{Experimental Protocol and Instructions}

Before the main experiment, each subject underwent a training run in the centrifuge. The onset angular acceleration and magnitude of the resultant gravitoinertial force were the same as in the main experiment. The subjects had, however, been given only very brief instructions to adjust the luminous line so that it looked horizontal; they had not been given any further information as to what was
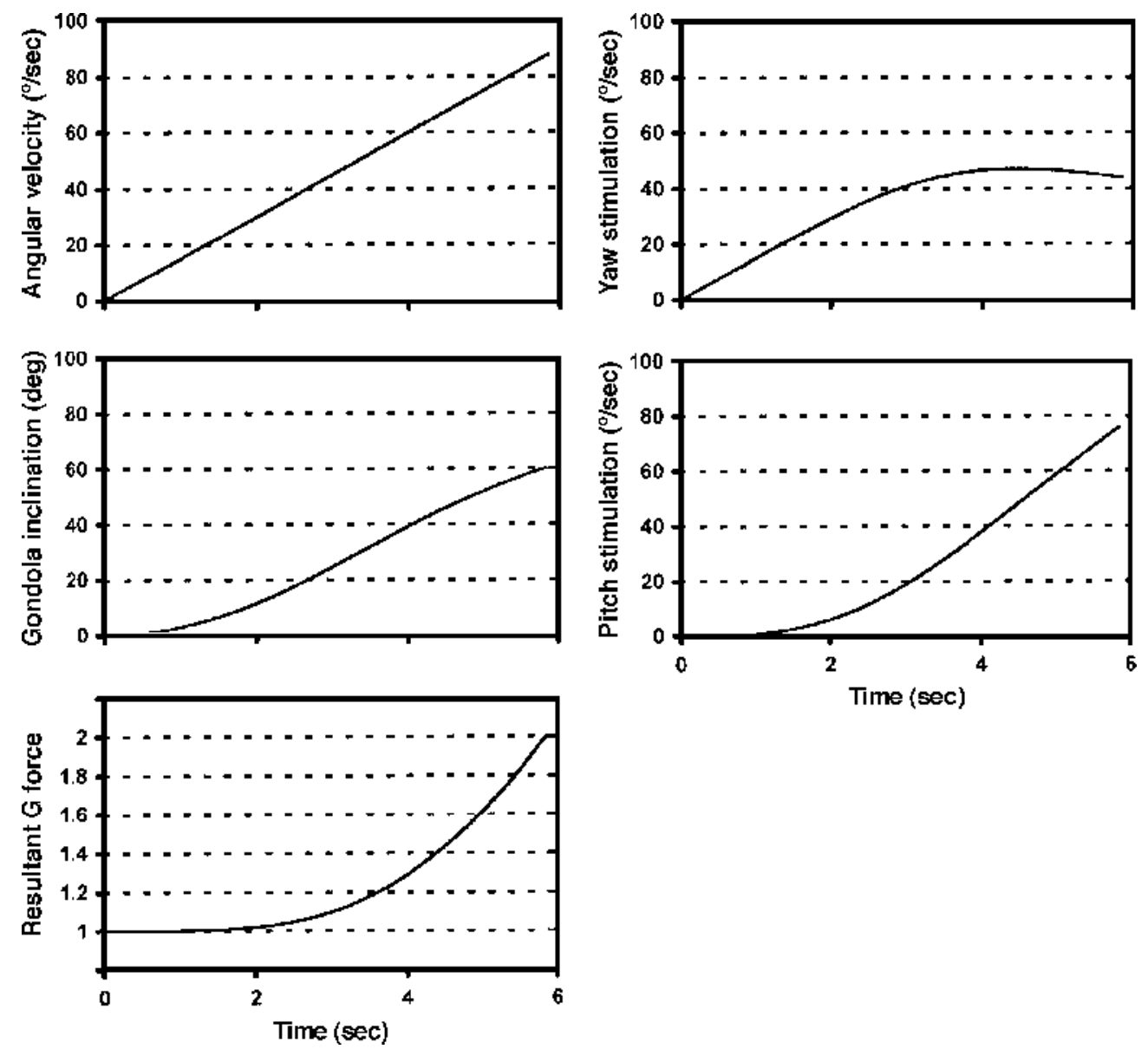

Figure 2. Diagrams showing the stimulus pattern during acceleration of the centrifuge. The yaw plane stimulus is the angular speed of the centrifuge $\times$ the cosine of the gondola inclination. The pitch plane stimulus is the angular speed of the centrifuge $\times$ the sine of the gondola inclination. 

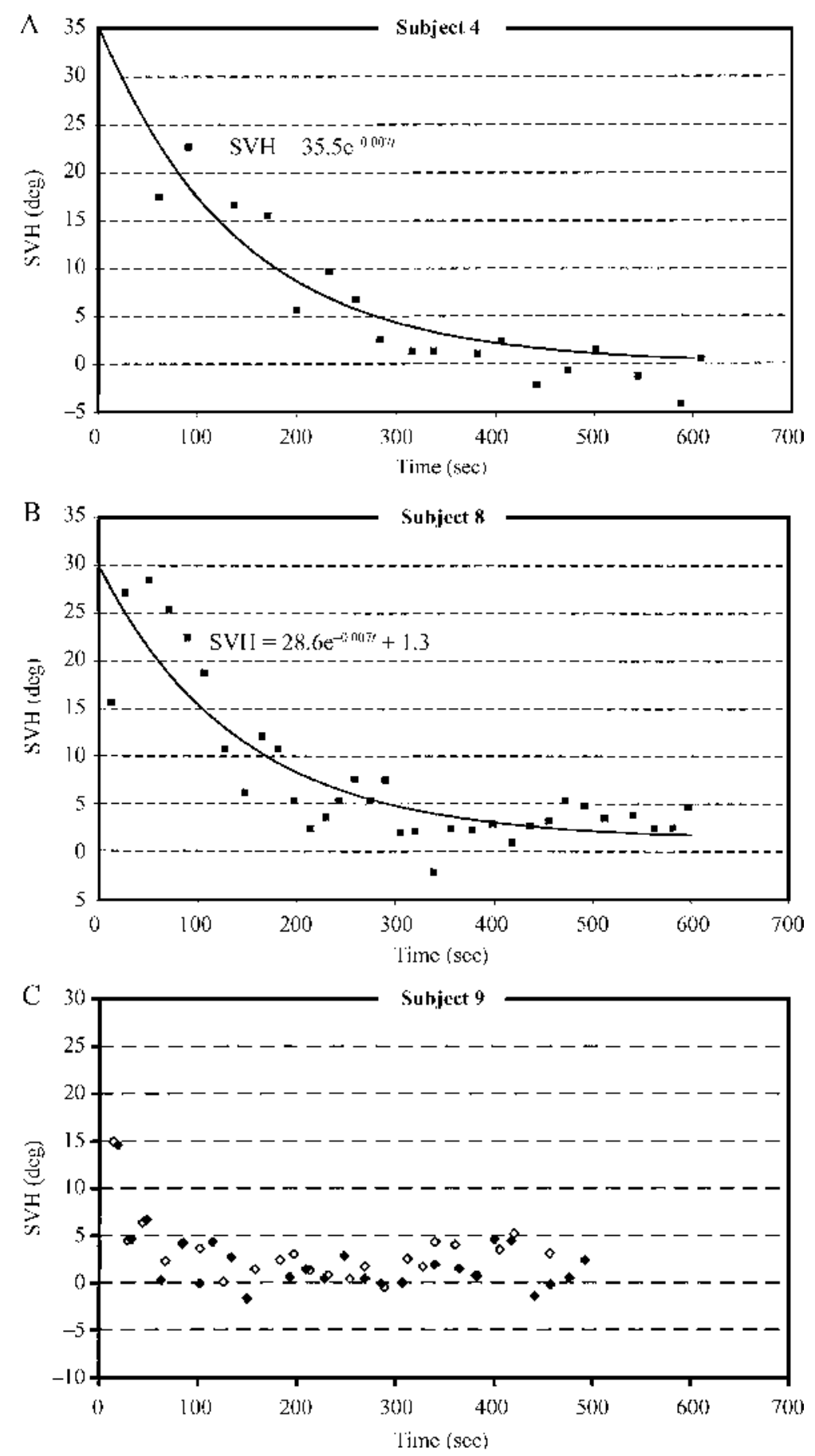

Figure 3A-C. Data from 3 subjects $(4,8$, and 9) indicating the SVH. Horizontal axis: time (in seconds) from the end of angular acceleration. Vertical axis: SVH (in degrees). Tilt to the right is denoted positive. For Subjects 4 and 8, the equations for best-fitting lines are presented. For Subject 9, the initial SVH tilt was reflected in only one setting of the line per centrifuge run. Therefore, reliable curve fitting could not be performed. Open and filled symbols represent data obtained during two separate runs.

meant by "horizontal." During this run, a few subjects spontaneously reported experiencing two possible horizontals, one coincident with the head "horizontal" (transversal) plane, the other representing an external horizon with respect to which the subject felt tilted. The for- mer is equivalent to the STP, whereas the latter is equivalent to the SVH. All subjects were interviewed after the training run about their experiences and strategies for adjusting the line. When confronted with the possibility of two different tasks, they were fully able to 
Table 1

Initial Tilts (in Degrees), Asymptotes (in Degrees), and

Time Constants (in Seconds) for Exponential Decay

\begin{tabular}{|c|c|c|c|c|c|c|c|}
\hline Subject & $\begin{array}{l}\text { Sequence } \\
\text { of Testing }\end{array}$ & $\begin{array}{c}\text { SVH } \\
\text { Initial } \\
\text { Tilt }\end{array}$ & $\begin{array}{c}\text { STP } \\
\text { Initial } \\
\text { Tilt }\end{array}$ & $\begin{array}{c}\text { SVH } \\
\text { Asymptote }\end{array}$ & $\begin{array}{c}\text { STP } \\
\text { Asymptote }\end{array}$ & $\begin{array}{c}\text { SVH } \\
\text { Time } \\
\text { Constant }\end{array}$ & $\begin{array}{c}\text { STP } \\
\text { Time } \\
\text { Constant }\end{array}$ \\
\hline 1 & $\mathrm{H}, \mathrm{T}, \mathrm{H}$ & +16.2 & -13.3 & -2.2 & -2.2 & 29 & 31 \\
\hline 2 & $\mathrm{H}, \mathrm{H}, \mathrm{H}, \mathrm{H}^{\mathrm{B}}$ & $+1.9^{\mathrm{A}}$ & B & $+1.9^{\mathrm{A}}$ & - & - & - \\
\hline 3 & $\mathrm{H}, \mathrm{H}, \mathrm{T}$ & +37.2 & -7.6 & +4.2 & +3.1 & 97 & 145 \\
\hline 4 & $\mathrm{H}, \mathrm{T}$ & +35.5 & $-5.7^{\mathrm{C}}$ & 0.0 & $-5.7^{\mathrm{C}}$ & 143 & - \\
\hline 5 & $\mathrm{~T}, \mathrm{H}, \mathrm{T}$ & +19.6 & $-6.9^{\mathrm{D}}$ & 0.0 & $-0.7^{\mathrm{E}}$ & 175 & - \\
\hline 6 & $\mathrm{H}, \mathrm{H}, \mathrm{T}$ & +7.4 & -15.2 & $+3.5^{\mathrm{F}}$ & -4.8 & $(1,000)^{\mathrm{F}}$ & 53 \\
\hline 7 & $\mathrm{~T}, \mathrm{H}$ & +7.5 & $(-2.1)^{\mathrm{G}}$ & 0.0 & $-0.9^{\mathrm{E}}$ & 625 & - \\
\hline 8 & $\mathrm{H}, \mathrm{T}, \mathrm{H}, \mathrm{T}$ & +22.2 & -16.0 & +4.6 & -5.9 & 142 & 45 \\
\hline 9 & H,T,H,T & $+14.8^{\mathrm{D}}$ & -8.9 & $+2.2^{\mathrm{E}}$ & -0.4 & - & 143 \\
\hline 10 & $\mathrm{H}, \mathrm{H}, \mathrm{T}, \mathrm{T}$ & +3.8 & -25.9 & +0.9 & +1.2 & - & 24 \\
\hline Mean & & +16.6 & -12.4 & +1.5 & -1.8 & 180 & 74 \\
\hline
\end{tabular}

Note- The SVH and STP were measured during different centrifuge runs, denoted by $\mathrm{H}$ or T. The values presented are based on data obtained during one or two runs. Each subject underwent two to four runs. AThe tilt of the SVH was very small from the beginning and did not show any tendency to change with time. Curve fitting gives a straight horizontal line corresponding to the mean of all data points. BSubject 2 was not able to imagine the head trans-

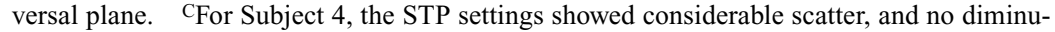
tion in tilt was apparent during the recording period. In such a case, the value for the initial tilt obtained via curve fitting corresponds to the mean of all settings performed during the registration period. The asymptote is the same as the estimated initial tilt. DFor the STP of Subject 5 and the SVH of Subject 9, the initial tilt was short lasting, and very few data points were obtained before the value was close to zero. The initial tilt has therefore been approximated with the mean of the first settings of two different $2 \mathrm{G}$ exposures. EMean of the data points with $t>300 \mathrm{sec}$. F Due to a slow decay and somewhat scattered data, it was hard to reliably estimate both the time constant and asymptote by means of curve fitting. The mean of the last 7 data points with $t>600 \mathrm{sec}$ was $+3.5^{\circ}$. Assuming the asymptote to be zero gives a time constant of $1,000 \mathrm{sec}$ (not included in group statistics). GDuring the first minute of centrifugation, Subject 7 was uncertain about how to perform the task. After discussions with the experimenter, she made four settings to the STP. These ranged from $-1.7^{\circ}$ to $-2.4^{\circ}$ without any tendency to change systematically with time. Since no measurements were made during the first minute, this mean value is not included in statistics on group level.

state which of them they had been performing. As a rule, after acceleration of the centrifuge the SVH was tilted to the right, whereas the STP was tilted to the left; the opposite was never the case.

In the main experiment, each subject underwent two to four centrifuge runs and was asked to consistently indicate either the SVH or the STP during each run. The instructions for SVH were as follows:

Imagine that you are sitting in a chair on a beach and looking out over the sea. Imagine the horizon of the sea and adjust the luminous line so that it is parallel with this horizon. If you have a sensation of being tilted sideways, then you should indicate the horizon in relation to which you feel tilted, not the transversal plane of your own head. Focus your attention on the line, not on your own body. Don't think to much, don't make any conscious calculation, we want to measure your spontaneous impression of the horizontal, not your ability to think.

The instructions for STP were the following:

Imagine the transversal plane of your head, or a line intersecting your eyes. Adjust the line so that it is parallel with this plane of your own irrespectively of the horizontal plane of the surroundings. If you feel tilted, then the line should also be set tilted.

In order to ensure a correct understanding of the task, the instructions were given in a dialogue with the subject. If there were any signs of uncertainty, the verbal instructions could be illustrated by gestures and head tilting of the experimenter.

Prior to centrifugation, when the subject had been positioned in the gondola, an initial series of eight settings of the luminous line to the SVH was performed. During the runs, data collection was started within a few seconds after the end of acceleration of the centrifuge and with few exceptions continued for at least $10 \mathrm{~min}$ at $2 \mathrm{G}$.

The SVV and SSP measurements were performed on a later occasion in order to confirm the findings of the first series of experiments. The procedure and instructions were similar, but the subjects were asked to set the line either as a plumb line (SVV) or parallel with the length axis of his/her own body (SSP).

\section{RESULTS}

\section{The SVH and SVV in $1 \mathrm{~g}$ Environment}

In the $1 \mathrm{~g}$ environment, all subjects except 1 had an SVH or SVV that was within the normal range $\left[-2.5^{\circ}\right.$ to $+2.5^{\circ}$ (Böhmer \& Rickenmann, 1995; Dai et al., 1989; Tribukait et al., 1996)]. The group mean of SVH was $-0.75^{\circ} \pm 1.1(S D), n=10$. For Subject 4 , there was a small tilt of the SVH to the left (M $\pm S D$ of eight settings: $\left.-2.9^{\circ} \pm 0.6^{\circ}\right)$. The group mean of the SVV was $-0.82^{\circ} \pm 0.57, n=5$.

\section{Perceptual Experiences and Principal Findings at 2G}

Acceleration of the centrifuge caused a sensation of head and body tilt to the left and induced considerable 
tilts in the settings of the line. At the $2 \mathrm{G}$ plateau, the SVH or SVV deviated to the right (clockwise from the subject's point of view) with respect to the resultant gravitoinertial horizontal or vertical. This deviation diminished during the registration period, often approaching zero within $10 \mathrm{~min}$. In contrast, when indicating the STP or SSP the luminous line was set tilted to the left (counterclockwise).

\section{Individual Data for SVH and STP at 2G}

Figures $3 \mathrm{~A}$ and $3 \mathrm{~B}$ show changes with time of the SVH for two subjects. In order to calculate the initial $\mathrm{SVH}$ tilts, an exponential function $\left(\mathrm{SVH}=\mathrm{Ae}^{-\mathrm{bt}}+\mathrm{C}\right)$ has been adapted to each series of data by means of a least-squares fit method. The initial tilt of the SVH is then obtained as $\mathrm{A}+\mathrm{C}$. The constant $\mathrm{C}$ denotes the asymptotic value approached by the $\mathrm{SVH}$; it was, as a rule, close to zero. The time constant for exponential decay is obtained as $\mathrm{T}=1 / \mathrm{b}$. For Subject 9 (see Figure $3 \mathrm{C}$ ), who had a rapid decay of the SVH tilt and for whom very few data points could be collected early during the $2 \mathrm{G}$ plateau, it was not possible to reliably estimate the initial tilt of the SVH by means of curve fitting. In this case, the initial tilt was approximated by the mean of the first data points of two separate centrifuge runs. However, no time constant was established. Regarding the STP, the time courses have been estimated in the same way as for the SVH. Data for all subjects are presented in Table 1.

As seen in Table 1, subjects were very different with respect to the magnitude of the SVH tilt. These differences did not seem to be random. Figures $4 \mathrm{~A}$ and $4 \mathrm{~B}$ show data from test and retest of Subjects 2 and 3 having very different initial values of the SVH. Similarly, Figures 5A and 5B show the test-retest data for the STP of Subjects 9 and 10. The reproducibility suggests that the interindividual differences were not simply due to poor precision in the settings of the luminous line. In addition, the individual pattern seemed to be unaffected by repeated testing.
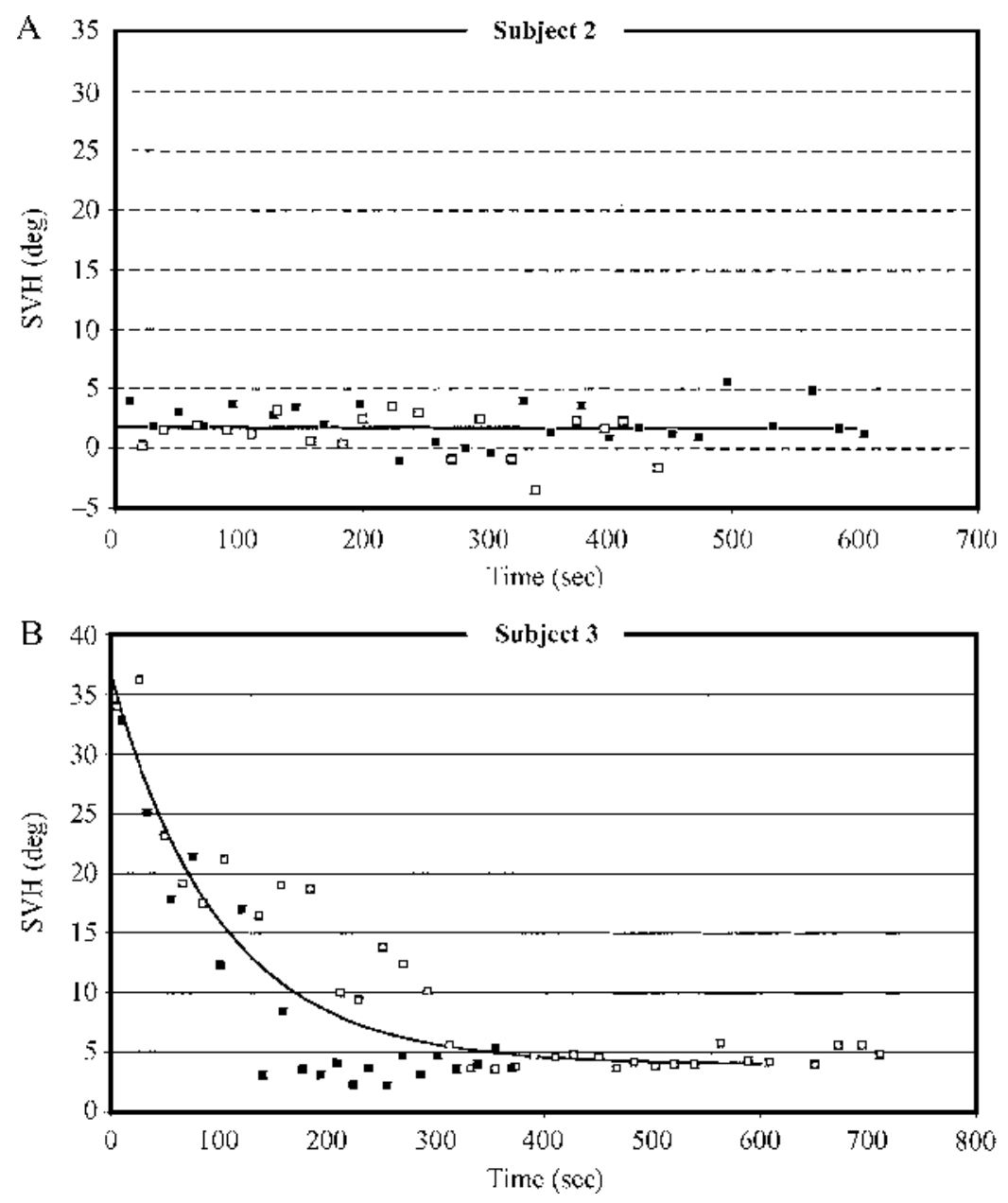

Figure 4. Test-retest data of Subjects 2 and 3 indicating the SVH. Horizontal axis: time (in seconds) from the end of angular acceleration. Vertical axis: SVH (in degrees). Tilt to the right is denoted positive. Open and filled symbols represent data obtained during two separate centrifuge runs. Best-fitting lines, as well as the values presented in Table 1, are based on both data series. 


\section{SVH and STP at 2G, Group Data}

Without exception, the allocentric task of indicating the SVH resulted in an initial SVH-tilt to the right, whereas the egocentric task of indicating the STP was associated with a tilt to the left. The initial tilt of the SVH was $+16.6^{\circ}$ $(M),+15.6^{\circ}$ (median), $S D=12.3^{\circ}$. The initial STP-tilt was $-12.4^{\circ}(M),-11.1^{\circ}$ (median), $S D=6.7^{\circ}$. The difference between the means for SVH and STP was highly significant (paired $t$ test; $p<.001, n=8$ ). However, regarding the absolute values for the means (16.6 and 12.4), there was no significant difference.

The asymptote for the SVH was $+1.5^{\circ}(M),+1.4^{\circ}$ (median), $S D=2.2^{\circ}$. The asymptote for the STP was $-1.8^{\circ}$ $(M),-0.9^{\circ}$ (median), $S D=3.1^{\circ}$. The difference between these means is significant (paired $t$ test; $p<.05, n=9$ ).

The time constant for exponential decay of the SVH tilt was $180 \mathrm{sec}(M), 142 \mathrm{sec}$ (median), $S D=203 \mathrm{sec}, n=7$. For the STP, the time constant was $74 \mathrm{sec}(M), 49 \mathrm{sec}$ (median); $S D=56 \mathrm{sec}, n=6$. Since both time constants could be calculated for only 4 subjects, it was not possible to establish whether there was any difference between them. For both tasks, there was a large range for the time constant.

\section{The SVV and SSP at $2 \mathrm{G}$}

Figure 6A, B shows changes with time of the SVV and SSP for Subjects 4 and 13. The pattern is the same as for the SVH and STP. Initial offsets and time constants for the 5 subjects are presented in Table 2. The initial tilt of the SVV was $+10.8^{\circ}(M), 9.4^{\circ}$ (median), $S D=5.8^{\circ}, n=5$. The initial tilt of the SSP was $-9.7^{\circ}(M),-10.1^{\circ}$ (median), $S D=6.1^{\circ}, n=4$. The difference between these two means was significant (paired $t$ test; $p<.01, n=5$ ).

The asymptote for the SVV was $+3.4^{\circ}(M),+1.1^{\circ}$ (median), $S D=4.9^{\circ}, n=5$. The asymptote for the SSP was $-2.2^{\circ}(M),-1.1^{\circ}$ (median), $S D=4.0^{\circ}, n=5$.

The time constant for the SVV was 134 seconds $(M)$, $118 \mathrm{sec}$ (median), $S D=109 \mathrm{sec}, n=3$. For the SSP, it was $104 \sec (M), 108 \mathrm{sec}$ (median), $S D=37, n=4$.
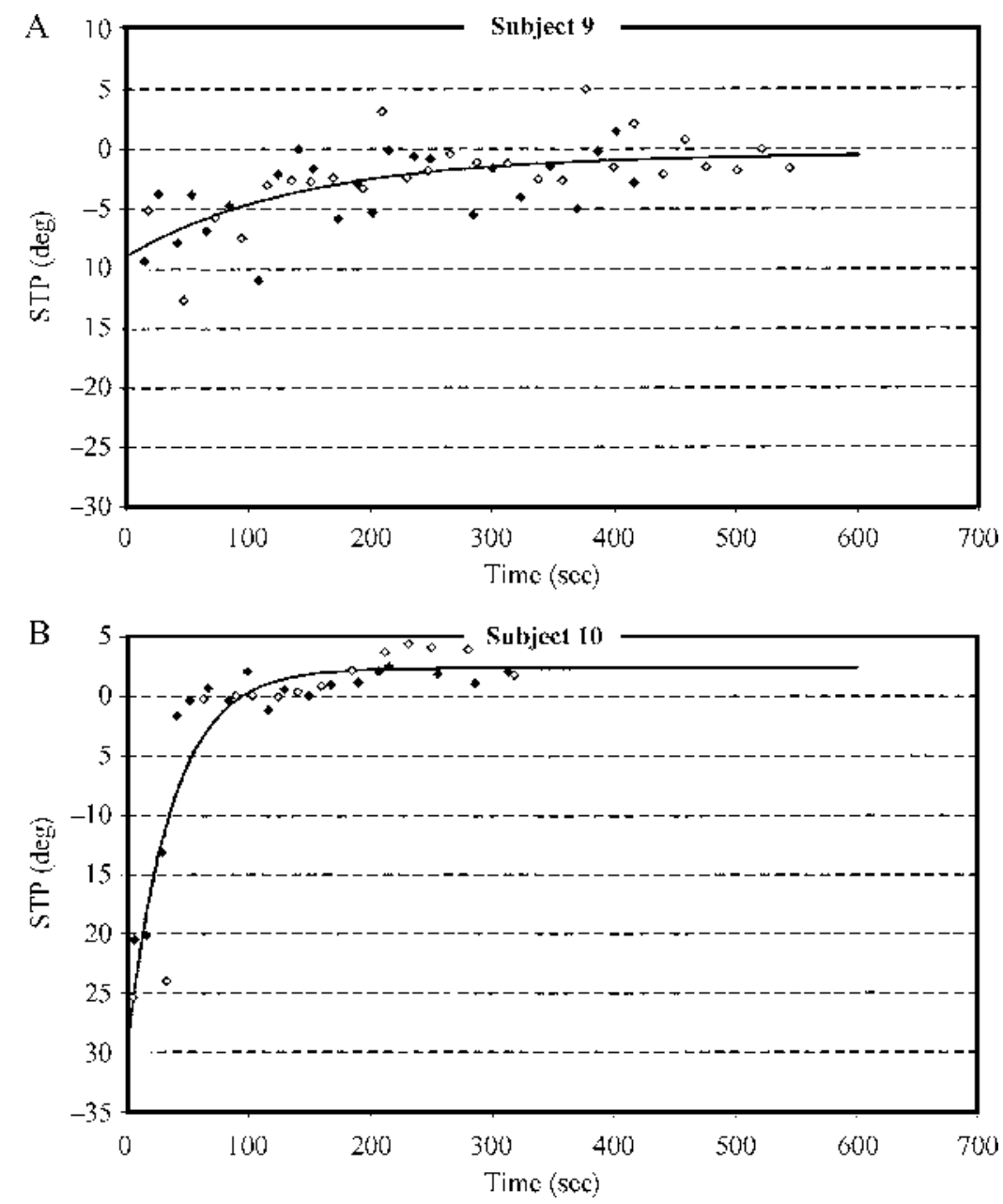

Figure 5. Test-retest data of Subjects 9 and 10 indicating the STP. Horizontal axis: time (in seconds) from the end of angular acceleration. Vertical axis: STP (in degrees). Negative sign denotes tilt to the left. Open and filled symbols represent data obtained during two separate centrifuge runs. Best-fitting lines, as well as the values presented in Table 1 , are based on both data series. 


\section{The Perceived Angle of Roll-Tilt}

An estimate of the subjects' perceived change in roll position at acceleration of the centrifuge can be obtained as the difference between the SVH and STP. For the 8 subjects who provided complete data series for both the SVH and STP, this difference was $32.0^{\circ}(M), 29.6^{\circ}$ (median), $S D=8.3^{\circ}, n=8$. The corresponding figures for the SVV or SSP were $20.5^{\circ}(M), 19.1^{\circ}$ (median), $S D=$ $7.4^{\circ}, n=5$. Combining the data gives $27.4^{\circ}(M), 26.5^{\circ}$ (median), $S D=9.7^{\circ}, n=11$.

\section{The SVH and SVV in Relation to the STP and SSP}

Figure 7 shows the initial tilts of the SVH and SVV (allocentric tasks) plotted against the initial tilts of the STP and SSP (egocentric tasks). There was no tendency for large tilts of the SVH or SVV to be associated with large tilts of the STP or SSP. Rather, the relationship tended to be reciprocal, although there was no significant correlation (linear regression; $r=.39, p=.23, n=11$ ).

\section{DISCUSSION}

\section{The Influence of Vestibular Stimulation on the SVH (or SVV) and on the Egocentric Frame of Reference}

The pivot of the gondola serves to maintain alignment of the subject's long $(z)$ axis, relative to the resultant gravitoinertial force vector (vectorial sum of the earth's gravity and the centrifugal force). Therefore, the otolith system does not receive any roll-tilt stimulus. However, during acceleration of the centrifuge there is a roll canal stimulus to the left caused by the swing out of the gondola and subject relative to the centrifuge main axis (Glasauer 1993; Guedry \& Oman, 1990; McGrath et al., 1995). In addition, there is a complex angular-velocity
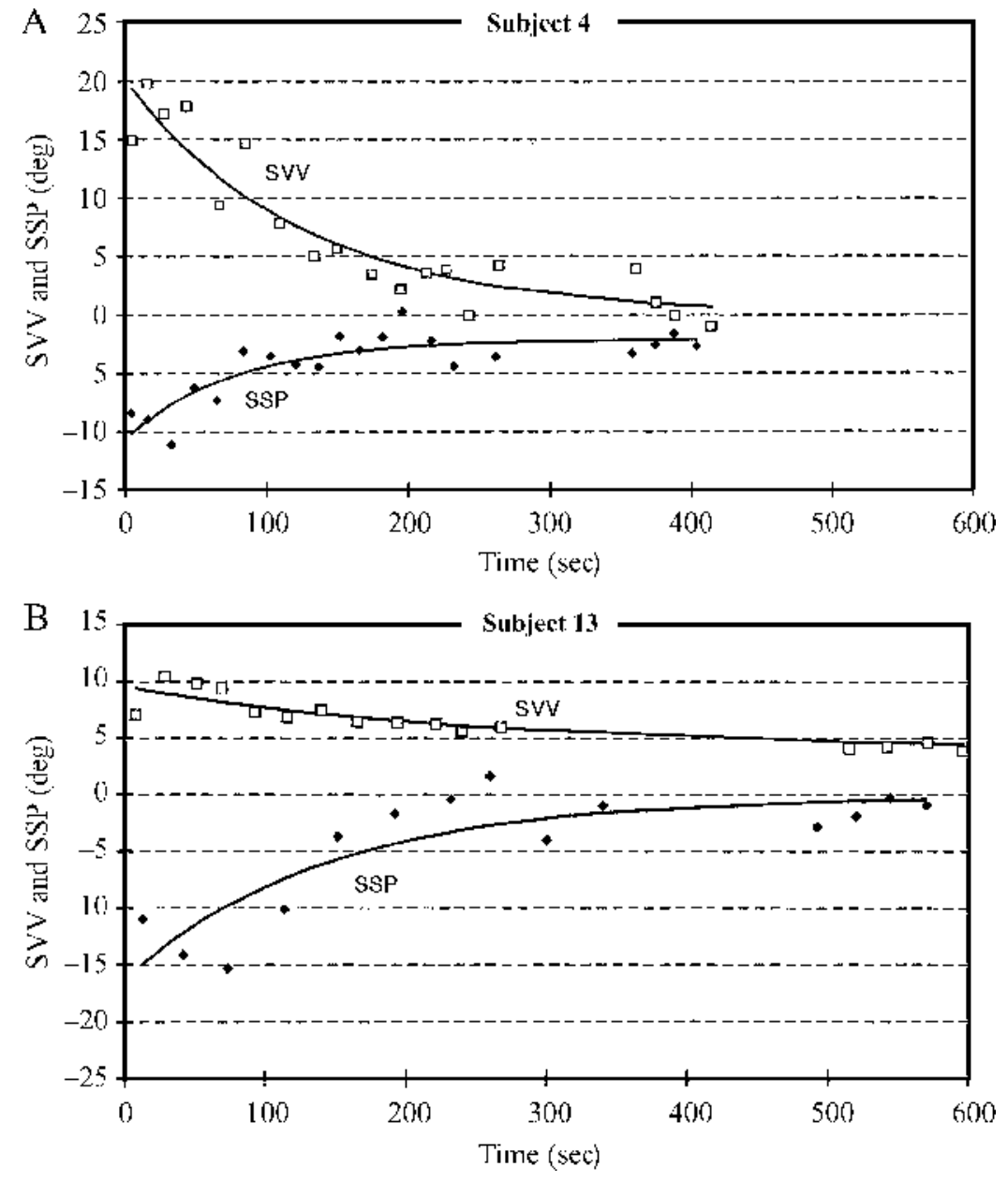

Figure 6. The SVV and SSP in Subjects 4 and 13. Horizontal axis: time (in seconds). Vertical axis: SSV/SSP (in degrees). Tilt to the right is denoted positive, and tilt to the left is denoted negative. For each subject, data have been collected during two separate runs. 
Table 2

Initial Tilts (in Degrees) and Time Constants (in Seconds)

for Exponential Decay of the SVV and SSP

\begin{tabular}{|c|c|c|c|c|c|c|c|}
\hline Subject & $\begin{array}{l}\text { Sequence } \\
\text { of Testing }\end{array}$ & $\begin{array}{c}\text { SVV } \\
\text { Initial } \\
\text { Tilt }\end{array}$ & $\begin{array}{c}\text { SSP } \\
\text { Initial } \\
\text { Tilt }\end{array}$ & $\begin{array}{c}\text { SVV } \\
\text { Asymptote }\end{array}$ & $\begin{array}{c}\text { SSP } \\
\text { Asymptote }\end{array}$ & $\begin{array}{c}\text { SVV } \\
\text { Time } \\
\text { Constant }\end{array}$ & $\begin{array}{c}\text { SSP } \\
\text { Time } \\
\text { Constant }\end{array}$ \\
\hline 4 & $\mathrm{~S}, \mathrm{~V}$ & +20.1 & -10.1 & 0.0 & -1.1 & 118 & 91 \\
\hline 9 & $\mathrm{~S}, \mathrm{~V}$ & +4.4 & -14.7 & +1.1 & -8.9 & 33 & 59 \\
\hline 11 & V,S & $+8.3^{\mathrm{A}}$ & -5.3 & $+0.3^{\mathrm{B}}$ & +1.3 & - & 143 \\
\hline 12 & V,S & $+11.7 \mathrm{C}$ & $-2.0^{\mathrm{C}}$ & $+11.7 \mathrm{C}$ & $-2.0^{\mathrm{C}}$ & - & - \\
\hline 13 & $\mathrm{~V}, \mathrm{~S}$ & +9.4 & -16.3 & +4.0 & -0.1 & 250 & 125 \\
\hline Mean & & +10.8 & -9.7 & +3.4 & -2.2 & 134 & 104 \\
\hline
\end{tabular}

Note-V, SVV; S, SSP. ABecause the decay was very rapid with few early data points, the initial tilt could not be reliably estimated by means of curve fitting. The mean of the first two data points is presented. BThe mean of the four last data points, obtained between minutes 4-6. CFor Subject 12, the SVV or SSP did not appear to change during the registration period. Therefore, the means of all data points are given and the asymptotes are equivalent with the initial offsets.

stimulus, gradually changing from the horizontal plane to near the pitch plane of the subject.

When the centrifuge has been accelerated up to $2 \mathrm{G}$, the roll change-in-position stimulus amounts to $60^{\circ}$. An angular displacement of this magnitude is, when performed within $6 \mathrm{sec}$, a stimulus well above the threshold of the semicircular canals. Furthermore, various studies have shown that normal subjects, undergoing angular displacements of this magnitude, may well estimate the magnitude of the rotation (Guedry, 1974; Guedry, Stockwell, Norman, \& Owens, 1971; Israël, Bronstein, Kanayama, Faldon, \& Gresty, 1996; Israël, Fetter, \& Koenig, 1993; Metcalfe \& Gresty, 1992), an ability that is dependent on central nervous integration of the semicircular canal signal over time (Baloh, 1989; Glasauer \& Mittelstaedt, 1998; Mergner, Rumberger, \& Becker, 1996).

If the subject were able to maintain a correct imagination of the external world, he or she would, at $2 \mathrm{G}$, set the

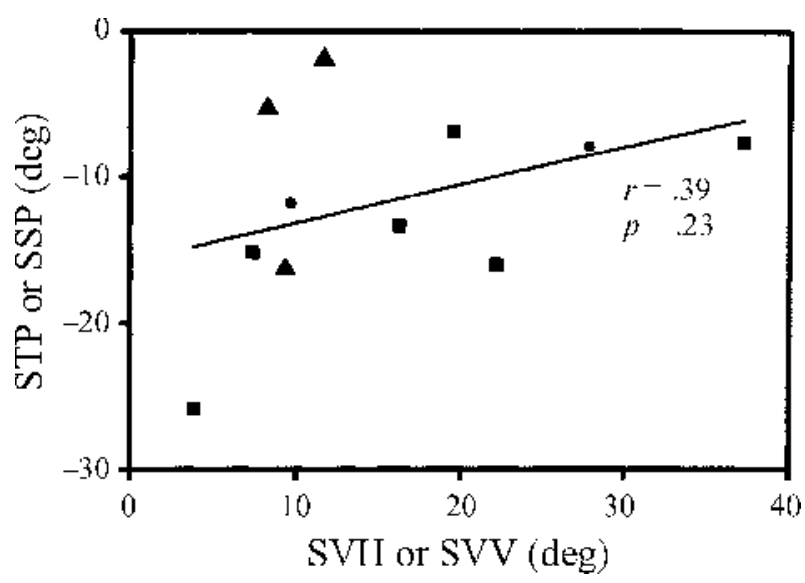

Figure 7. Relationship between the SVH and SVV and between the STP and SSP. Tilt to the right is denoted positive. Linear regression: $r=.39, p=.23, n=11$. Squares represent $\mathrm{SVH}$ and STP. Triangles represent SVV and SSP. For Subjects 4 and 9, there were data for both the SVH and SVV, as well as for the STP and SSP. The means of these are represented by circles. luminous line tilted by $60^{\circ}$ to the right, which is also to be expected of a perfect acceleration processor (Holly, 2000). However, in the present study the initial tilt of the SVH was, on average, only $16.6^{\circ}$. For the SVV, it was $10.8^{\circ}$. These figures correspond to a gain for the perception of roll inclination of $28 \%$ and $18 \%$ for $\mathrm{SVH}$ and SVV, respectively. The cause of this underestimation of roll, as measured by settings of a luminous line, has been suggested to be the lack of otolithic signal for head tilt (Glasauer \& Mittelstaedt, 1998) or, alternatively, the persistent otolithic signal for upright position counteracting the semicircular canal signal for lateral tilt (Tribukait, 1999).

The egocentric task of indicating the orientation of the transversal or sagittal planes of the head (STP or SSP) has a quite different relationship to vestibular stimulation than has the allocentric task of indicating the subjective horizontal or vertical of the surroundings ( $\mathrm{SVH}$ or SVV). Adjusting a line so that it is parallel with a head transversal plane may, in principle, be adequately done without any idea about the orientation of one's own body with respect to the surroundings. The prerequisites are just that the subject must have an awareness of the polarity of his or her own head and body and that the visual system correctly estimates the orientation of the line with respect to the head. Thus, such an egocentric orientation task should be largely independent of the vestibular organs. In fact, it has been demonstrated that healthy subjects may correctly indicate the orientation of their own length axis even in positions where the imagination about the gravitational vertical is erroneous by $20^{\circ}-30^{\circ}$ (Haustein, 1992; H. Mittelstaedt, 1983). Against the background of such facts, the tilt of the STP and SSP in the present study seems quite remarkable.

There seem to be two principally different ways of explaining the deviation of the STP and SSP. One primarily concerns visual functions, and the other is related to the imagination about the polarity of one's own body - that is, the egocentric frame of reference. Regarding the former, a distortion of visual space may be induced by 
changes in ocular torsional position that are not adequately accounted for by the brain. However, a mere change in eye position seems very unlikely to cause the tilt of the STP or SSP. A stimulus that is symmetric - that is, the same to the otolith organs on both sides - would not cause any systematic change in the torsional position of the eye. Furthermore, the semicircular canal signal at a head tilt to the left would cause rightward eye torsionthat is, upper poles toward the right shoulder (Petrov \& Zenkin, 1973) - and, in addition, tilts of the SVH correspond in direction to changes in torsional eye position (Curthoys, Dai, \& Halmagyi, 1991; Wade \& Curthoys, 1997). Another possible factor is a change in ocular torsional position induced by the yaw-plane angular-velocity stimulus. However, yaw-left rotation causes the eyes to roll with the upper pole toward the right shoulder (Wade \& Curthoys, 1997). These facts suggest that any change in torsional position of the eyes would contribute more likely to the rightward tilt of the SVH or SVV than to the leftward tilt of the STP and SSP. Thus, the leftward tilt of the STP or SSP cannot be explained by ocular torsion.

A slightly different possibility is related to efference copies (von Holst \& Mittelstaedt, 1950). If the efference copy - a kind of memory of a motor command to the eye muscles - corresponds to a larger torsion than the actual torsion, there would be a change in the visually perceived horizontal or vertical in the opposite direction of the torsion. However, the findings by Wade and Curthoys (1997) suggest that vestibularly induced ocular torsion is not accompanied by efference copies; in all subjects, the torsion corresponded with an SVH-tilt in the same direction. Furthermore, if the rightward tilt of the SVH or SVV is regarded as the effect of a distortion of visual space, the leftward tilt of the STP or SSP should not be given a similar interpretation, since one must then assume the existence of more than one visual space (or a "splitting" of visual space), which seems to conflict with the basic principle known as Ockham's razor.

Therefore, what has to be especially considered is the mental representation of one's own spatial extension and polarity, involving a coordinate system that is fixed with respect to the head and body. As mentioned in the introduction, caloric irrigation may induce a shift about the head-vertical axis of the egocentric frame of reference. Vestibular stimulation during acceleration of the centrifuge seems to influence this fundamental frame of reference so that the imagined transversal and sagittal head planes are tilted to the left with respect to the physical head planes. This would cause a tilt of the STP or SSP even if there were no deviation of the eye with respect to the head.

In our experiments, the STP did not seem to be a simple inversion of the SVH. Rather, a large deviation in the STP tended to be associated with a small tilt of the SVH, suggesting that a change in the mental representation of the body may influence the measure of one's own orientation with respect to the surroundings. Therefore, deviations of the mental representation of the body length axis or transversal plane also have consequences for the interpretation of the SVH and SVV. The adequate measure of perceived change in roll position after acceleration of the centrifuge would not simply be the value for the SVH or SVV but rather the difference between the SVH and STP or between the SVV and SSP. The data obtained in the present study would imply a perceived change in roll position of $27^{\circ}$ and, thus, a gain of $45 \%$.

According to the theories on spatial orientation in weightlessness, which have been developed by Glasauer and Mittelstaedt (1998), the measure of roll-tilt perception should be very small when the semicircular canal message on a change in roll position is not accompanied by any corresponding signal from the otolith organs. A possible explanation for the discrepancy between this theory and the present results obtained in hypergravity may be found in the pattern of stimulation during acceleration of the centrifuge. This is very different from the stimulus at a roll head movement in weightlessness. Besides the roll change-in-position stimulus to the vertical canals, there is a complex angular-velocity stimulus, gradually changing from the horizontal plane to near the pitch plane of the subject. This pattern of angular-velocity stimulation is quite specific for entering a coordinated bank and turn, such as, for instance, when flying an aircraft or driving a motorcycle, but also when running. Thus, since this complex of angular-velocity stimulation is likely to occur mainly when there is also a change in roll position, it would reasonably be interpreted by the brain as an additional message about the change in roll position.

Another interesting phenomenon, also suggesting that the angular-velocity stimulus may contribute to changes in the SVH, is the so-called oculo-gravic lag effect during eccentric rotation in a fixed chair centrifuge (Graybiel \& Brown, 1951; Guedry, 1974). It has been demonstrated that this delay in reorientation to the tilted gravitoinertial force vector is caused by the yaw plane canal stimulus during acceleration (Holly 1997; Merfeld, Zupan, \& Gifford, 2001).

An earlier study (Tribukait, 2003), in which curve fitting was performed to data obtained at $1.1-1.3 \mathrm{G}$, suggests that the tilt of the SVH/SVV, induced by acceleration of the centrifuge, depends on two different mechanisms related to semicircular canal stimulation. Namely, there is a large component of the SVH-tilt, decaying with a time constant of 2-3 min and a small component (a few degrees) that is very long lasting. It might be hypothesized that the former is related to the complex angular-velocity stimulation and the latter to the roll change-in-position stimulus caused by the inclination of the gondola. The small component (the asymptote $\mathrm{C}$ ) is well in accordance with the theories of Glasauer and Mittelstaedt (1998). In the present study, the difference between the asymptotic values for the SVH or SVV, on the one hand, and the STP or SSP, on the other hand, was in the order of $3^{\circ}-6^{\circ}$.

\section{Interindividual Variability}

Regarding the magnitude of tilt of the SVH and SVV at $2 \mathrm{G}$, there were considerable differences between sub- 
jects. A possible explanation is an interindividual variability in the weighting of input from the otolith organs and the semicircular canals. When changing the head's position with respect to gravity at $1 \mathrm{~g}$, the information provided by the two different receptor systems will be redundant. Therefore, any preference for one or the other type of sensory information will not make any difference for the perception of the magnitude of angular head movements with respect to gravity. However, in case of a conflict between the otolith organs and the semicircular canals, the perceptual outcome will depend on whether the individual relies comparatively more on otolithic or semicircular canal information about changes in head position. After acceleration in a gondola centrifuge, the tilts of SVH or SVV in semicircular dependent subjects will be large, whereas those who depend primarily on otolithic information (such as possibly Subject 2 in the present study) will show small tilts of the SVH or SVV and small gains for the perception of roll inclination.

Another explanation for the great interindividual variability found in the present study, as well as in earlier ones (Tribukait, 1999, 2003), could be that subjects used different strategies when trying to indicate the SVH. An interesting case is Subject 2 of the present study. During four different centrifuge runs, he showed an SVH that was close to zero. Furthermore, he stated that he was not able to imagine his own head transversal plane. If this subject were really indicating the STP, believing that it was the SVH, the task to indicate the STP must have seemed very strange to him. However, Subject 2 stated that he had almost no sensation of being tilted after acceleration of the centrifuge. In addition, the small testretest variability found within subjects, who had been given the same thorough instructions, suggests that the interindividual variability is to a considerable extent related to some preconscious mechanisms and not only to differences on a psychological level. In addition, subjects did not divide naturally into subgroups, as might be expected in the case of two or three strategies/tasks, each with a small interindividual variability. Rather, the initial SVH tilts seemed to range over a continuous spectrum.

In addition, an interindividual variability is a prerequisite for the study of covariance or independence of test variables. The SVH or SVV and STP or SSP seemed quite independent regarding the magnitude of initial tilt. If this is not simply due to noise, it deserves an explanation. One possibility is that the SVH or SVV and STP or SSP depend on different sensory information. It has been suggested by Mittelstaedt that while both the SVV and SSP depend on the vestibular organs, the SVV is also influenced by the idiotropic vector (H. Mittelstaedt, 1995b), whereas the SSP to some extent depends on the gravity receptors in the abdomen (H. Mittelstaedt, 1995a).

\section{Different Memories for Semicircular Canal Information}

During constant speed rotation, the activity of semicircular canal nerve afferents decays exponentially with a time constant of 2-6 sec (see Goldberg \& Fernandez, 1984 , for references), without any difference between the horizontal and vertical canals. However, the compensatory (slow) phase of rhythmic eye movements (nystagmus), which serves to stabilize the gaze with respect to the surroundings, has been found to decay with a time constant of 15-20 sec for the horizontal canals; for vertical canal stimulation, the time constant is half of that (see Young, 1984, for references). This increase of the time constant is ascribed to a central nervous function (Baloh, 1989; Young, 1984) often called the velocity-storage mechanism (Raphan, Matsuo, \& Cohen, 1979), which is a kind of working memory increasing the time during which eye velocity corresponds to head velocity. This velocity-storage mechanism may also influence the duration of certain spatial illusions. It has, for instance, been found that subjects, seated upright and rotated about a head-centered earth-vertical axis, have a tilt of the SVV (Wapner, Werner, \& Morant, 1951) or SVH (Wade \& Curthoys, 1997). In the study by Wade and Curthoys, the magnitude of this tilt was $2^{\circ}-10^{\circ}$ initially at an angular velocity of $250 \% \mathrm{sec}$, and it ceased completely within $30-40 \mathrm{sec}$ of constant velocity. This is compatible with the time constant for the velocity-storage mechanism - that is, 10-15 sec (after three $\times$ the time constant, an exponentially decaying phenomenon approximates the baseline).

In addition to dynamic responses during angular movements, the semicircular canal system is involved in sensing angular changes in position (Guedry, 1974; Israël et al., 1996; Israël et al., 1993; Metcalfe \& Gresty, 1992). This function depends on central nervous integration of the semicircular canal velocity signal over time (Baloh, 1989) — that is, a "velocity-to-position integrator" (Glasauer \& Mittelstaedt, 1998; Mergner et al., 1996). The usefulness of such information on changes in head position must depend on memory functions, especially when there is some latency between a perceived change in angular position and the execution of a spatial orientation task, as well as during such orientation tasks which in themselves have a considerable duration-for example, navigation by path integration (H. Mittelstaedt \& M.-L. Mittelstaedt, 1982).

During gondola centrifuging, the time constant for exponential decay of the SVH or SVV tilt is considerably larger than that for phenomena related to the velocitystorage mechanism. The decay of the SVH or SVV tilt during gondola centrifuging reflects the persistence of a memory of the change in roll position; a smaller time constant implies more rapid forgetting. This memory function, or "position-storage mechanism," which has been treated more extensively in an earlier paper (Tribukait, 2003), implies that the effects of a small and short-lasting semicircular canal stimulus may have a much larger duration than what is usually believed. It might be speculated whether the position-storage mechanism exerts its influence by prolonging a side difference induced in the central vestibular system by a semicircular canal stimulus. 
To measure the SVH or SVV during gondola centrifuging seems to be a useful method for obtaining quantitative data on vestibular memory functions in humans, for whom neuronal mechanisms underlying the mental representation of external space, involving so-called place cells and head direction cells (for a review, see Taube, 1998), cannot be directly studied.

It is to be noted, in this context, that there are other situations in which the time constant of a vestibular phenomenon is larger than that of the velocity-storage function. Subjects walking blindfolded in a circle and asked to keep a constant speed show an exponential decline in angular velocity. The time constant for this decline increases linearly with the magnitude of centripetal acceleration, approximately $50 \mathrm{sec}$ at $1.2 \mathrm{~m} / \mathrm{sec}^{2}$ (M.-L. Mittelstaedt \& Jensen, 1999; M.-L. Mittelstaedt \& H. Mittelstaedt, 1997). Thus, there is the possibility that position storage as well depends to some extent on the magnitude of the resultant gravitoinertial force. Further studies in the centrifuge will be required in order to elucidate this matter.

\section{Practical Implications}

The memory of the semicircular canal system for angular head movements must be accounted for when one is attempting to study, for instance, how changes in head position relative to gravity are detected by the otolith organs. If a change in head position is detected by the semicircular canals, reliable data on otolithic function cannot be obtained until after several minutes.

Furthermore, quantitative measurement of the degree and duration of the effects of vestibular stimulation on the perception of the horizontal or vertical constitutes a means for understanding the mechanisms for spatial disorientation in aviators, which has caused many fatal accidents. A pioneering experiment was performed in the 1920 s by A. Tschermak and G. Schubert, who measured the subjective visual vertical during curve flight (Tschermak \& Schubert, 1931). At $2 \mathrm{G}$ and $60^{\circ}$ of roll, the single subject (one of the authors) indicated a tilt of the subjective vertical that was $10^{\circ}-15^{\circ}$ opposite to the roll of the airplane. Interestingly, he also indicated the subjective direction of the body length axis, which was tilted approximately $10^{\circ}$ in the other direction. Although these values were based on a few measurements in a single individual and not explained in vestibular terms, they are consistent with the findings of the present work.

One of the most common illusions when cloudy weather or darkness compromises vision consists in an erroneous sensation of roll attitude - the leans (Benson, 1999). The present data suggest that normal subjects may be very different in their abilities to estimate changes in roll position when there is a conflict between the semicircular canals and otolith organs. The nature of this interindividual variability warrants further investigation. The memory of the semicircular canal system for changes in head position is another important factor. This position-storage mechanism can probably assure adequate orientation in some situations, but it may also increase the duration of illusions like the leans.

\section{REFERENCES}

BALOH, R. W. (1989). Vestibulo-ocular, cervico-ocular, and visuoocular reflexes. Bulletin de la Societé Belge d'Ophtalmologie, 237, 35-66.

Benson, A. J. (1999). Spatial disorientation-common illusions. In J. Ernsting, A. N. Nicholson, \& D. J. Rainford (Eds.), Aviation medicine. Oxford: Butterworth \& Heinemann.

BöHMER, A., \& RicKENMANN, J. (1995). The subjective visual vertical as a clinical parameter of vestibular function in peripheral vestibular diseases. Journal of Vestibular Research, 5, 35-45.

Bottini, G., Karnath, H.-O., Vallar, G., Sterzi, R., Frith, C. D., Frackowiak, R. S. J., \& Paulesu, E. (2001). Cerebral representations for egocentric space: Functional-anatomical evidence from caloric vestibular stimulation and neck vibration. Brain, 124, 1182-1196.

Brandt, T., \& Dieterich, M. (1987). Pathological eye-head coordination in roll: Tonic ocular tilt reaction in mesencephalic and medullary lesions. Brain, 110, 649-666.

Curthoys, I. S., Dai, M. J., \& Halmagyi, G. M. (1991). Human ocular torsional position before and after unilateral vestibular neurectomy. Experimental Brain Research, 85, 218-225.

Dai, M. J., Curthoys, I. S., \& Halmagyi, G. M. (1989). Linear acceleration perception in the roll plane before and after unilateral vestibular neurectomy. Experimental Brain Research, 77, 315-328.

Glasauer, S. (1993) Human spatial orientation during centrifuge experiments: Non-linear interaction of semicircular canals and otoliths. In H. Krejcova \& J. Jerabek (Eds.), Proceedings of the XVIIth Barany Society Meeting (pp. 48-52). Prague.

Glasauer, S., \& Mittelstaedt, H. (1998). Perception of spatial orientation in microgravity. Brain Research \& Brain Research Reviews, 28, 185-193.

Goldberg, J. M., \& Fernandez, C. (1984). The vestibular system. In J. M. Brookhart \& V. B. Mountcastle (Series Eds.) \& I. Darian-Smith \& S. R. Geiger (Vol. Eds.), Handbook of physiology: Section 1. The nervous system: Vol. III. Sensory processes. Bethesda, MD: American Physiological Society.

Graybiel, A., \& Brown, R. H. (1951). The delay in visual reorientation following exposure to a change in direction of resultant force on a human centrifuge. Journal of General Psychology, 45, 143-150.

Guedry, F. E. (1974). Psychophysics of vestibular sensation. In H. H. Kornhuber (Series Ed.) \& D. Bagger-Sjöbäck et al. (Vol. Eds.), Handbook of sensory physiology: Vol. VI. Vestibular system: Pt. 2. Psychophysics, applied aspects, and general interpretation. Berlin: SpringerVerlag.

GuEdRY, F. E., \& OMAN, C. M. (1990). Vestibular stimulation during a simple centrifuge run (NAMRL 1353). Pensacola, FL: Naval Aerospace Medical Research Laboratory.

Guedry, F. E., Stockwell, C. W., Norman, J. W., \& Owens, G. G. (1971). Use of triangular waveforms of angular velocity in the study of vestibular function. Acta Oto-Laryngologica, 71, 439-448.

HAusteIN, W. (1992). Head-centric visual localization with lateral body tilt. Vision Research, 32, 669-673.

Holdstock, J. S., Mayes, A. R., Cezayirli, E., Isaac, C. L., AggleTON, J. P., \& ROBERTS, N. (2000). A comparison of egocentric and allocentric spatial memory in a patient with selective hippocampal damage. Neuropsychologia, 38, 410-425.

HoLLY, J. E. (1997). Three-dimensional baselines for perceived selfmotion during acceleration and deceleration in a centrifuge. Journal of Vestibular Research, 7, 45-61.

HoLLy, J. E. (2000). Baselines for three-dimensional perception of combined linear and angular self-motion with changing rotational axis. Journal of Vestibular Research, 10, 163-178.

HowARD, I. P., \& TEMPLETON, W. B. (1966). Human spatial orientation. London: Wiley.

Israël, I., Bronstein, A. M., Kanayama, R., Faldon, M., \& GRESTY, M. A. (1996). Visual and vestibular factors influencing vestibular navigation. Experimental Brain Research, 112, 411-419.

IsRaËL, I., FetTer, M., \& Koenig, E. (1993). Vestibular perception of passive whole-body rotation about horizontal and vertical axes in humans: Goal-directed vestibulo-ocular reflex and vestibular memorycontingent saccades. Experimental Brain Research, 96, 335-346. KARNATH, H.-O. (1997). Spatial orientation and the representation of 
space with parietal lobe lesions. Philosophical Transactions of the Royal Society of London: Series B, 352, 1411-1419.

McGrath, B. J., Guedry, F. E., Oman, C. M., \& Rupert, A. H. (1995). Vestibulo-ocular response of human subjects in a pivoting support system during $3 \mathrm{Gz}$ centrifuge stimulation. Journal of Vestibular Research, 5, 331-347.

Merfeld, D. M., Zupan, L. H., \& Gifford, C. A. (2001). Neural processing of gravito-inertial cues in humans: II. Influence of the semicircular canals during eccentric rotation. Journal of Neurophysiology, 85, 1648-1660.

Mergner, T., Rumberger, A., \& Becker, W. (1996). Is perceived angular displacement the time integral of perceived angular velocity? Brain Research Bulletin, 40, 467-471.

MetCalfe, T., \& GResty, M. (1992). Self-controlled reorienting movements in response to rotational displacements in normal subjects and patients with labyrinthine disease. Annals of the New York Academy of Sciences, 656, 695-698.

Mittelstaedt, H. (1983). A new solution to the problem of the subjective vertical. Naturwissenschaften, 70, 272-281.

Mittelstaedt, H. (1991). The role of the otoliths in the perception of the orientation of self and world to the vertical. Zoologische JahrbücherAbteilung für allgemeine Zoologie und Physiologie der Tiere, 95, 419425.

Mittelstaedt, H. (1995a). The formation of the visual and the postural vertical. In T. Mergner \& F. Hlavacka (Eds.), Multisensory control of posture (pp. 147-155). New York: Plenum.

MitTelstaedT, H. (1995b). New diagnostic tests for the function of utricles, saccules, and somatic graviceptors. Acta Oto-Laryngologica, 115 (Suppl. 520), 188-193.

Mittelstaedt, H., \& Fricke, E. (1988). The relative effect of saccular and somatosensory information on spatial perception and control. Advances in Oto-Rhino-Laryngology, 42, 24-30.

Mittelstaedt, H., \& Mittelstaedt, M.-L. (1982). Homing by path integration. In F. Papi \& H. G. Wallraff (Eds.) Avian navigation (pp. 290-297). Berlin: Springer-Verlag.

Mittelstaedt, M.-L., \& Jensen, W. (1999). Centrifugal force affects perception but not nystagmus in passive rotation. In B. Cohen \& B. J. M. Hess (Eds.), Otolith function in spatial orientation and movement (Annals of the New York Academy of Sciences, Vol. 871, pp. 435-438). New York: New York Academy of Sciences.

Mittelstaedt, M.-L., \& Mittelstaedt, H. (1997). The effect of centrifugal force on the perception of rotation about a vertical axis. Naturwissenschaften, 84, 366-369.

Petrov. A. P., \& Zenkin, G. M. (1973). Torsional eye movements and constancy of the visual field. Vision Research, 13, 2465-2477.

Raphan, T., Matsuo, V., \& Cohen, B. (1979). Velocity storage in the vestibulo-ocular reflex arc (VOR). Experimental Brain Research, 35, 229-248.

Rorden, C., Karnath, H.-O., \& Driver, J. (2001). Do neck-proprio- ceptive and caloric-vestibular stimulation influence covert visual attention in normals, as they influence visual neglect? Neuropsychologia, 39, 364-375.

SCHÖNE, H. (1964). On the role of gravity in human spatial orientation. Aerospace Medicine, 35, 764-772.

Stockwell, C. W., \& Guedry, F. E. (1970). The effects of semicircular canal stimulation during tilting on the subsequent perception of the visual vertical. Acta Oto-Laryngologica, 70, 170-175.

TAUBE, J.S. (1998). Head direction cells and the neurophysiological basis for a sense of direction. Progress in Neurobiology, 55, 225-256.

Tribukait, A. (1999). Semicircular canal and saccular influence on the subjective visual horizontal during gondola centrifugation. Journal of Vestibular Research, 9, 347-357.

TribuKaIT, A. (2003). Human vestibular memory studied via measurement of the subjective horizontal during gondola centrifugation. Neurobiology of Learning \& Memory, 80, 1-10.

Tribukait, A., Bergenius, J., \& Brantberg, K. (1996). The subjective visual horizontal for different body tilts in the roll plane: Characterization of normal subjects. Brain Research Bulletin, 40, 375-383.

Tribukait, A., Bergenius, J., \& Brantberg, K. (1998). Subjective visual horizontal during follow-up after unilateral vestibular deafferentation with gentamicin. Acta Oto-Laryngologica, 118, 479-487.

Tschermak, A., \& SCHubert, G. (1931). Über Vertikalorientierung im Rotatorium und im Flugzeuge. Pflügers Archiv für die gesamte Physiologie des Menschen und der Tiere, 228, 234-257.

Udo de Haes, H A. (1970). Stability of apparent vertical and ocular countertorsion as a function of lateral tilt. Perception \& Psychophysics, 8, 137-142.

Vallar, G., Lobel, E., Galati, G., Berthoz, A., Pizzamiglio, L., \& Le Bihan, D. (1999). A fronto-parietal system for computing the egocentric spatial frame of reference in humans. Experimental Brain Research, 124, 281-286.

von Holst, E., \& Mittelstaedt, H. (1950). Das Reafferenzprinzip. Naturwissenschaften, 37, 265-272.

WAdE, S. W., \& CuRThoys, I. S. (1997). The effect of ocular torsional position on perception of the roll tilt of visual stimuli. Vision Research, 37, 1071-1078.

WAPNER, S., Werner, H., \& Morant, R. B. (1951). Experiments on sensory-tonic field theory of perception: III. Effects of body rotation on the visual perception of verticality. Journal of Experimental Psychology, 42, 351-357.

YounG, L. R. (1984). Perception of the body in space: Mechanisms. In J. M. Brookhart \& V. B. Mountcastle (Series Eds.) \& I. Darian-Smith \& S. R. Geiger (Vol. Eds.), Handbook of physiology: Section 1. The nervous system: Vol. III. Sensory processes. Bethesda, MD: American Physiological Society.

(Manuscript received May 15, 2003; revision accepted for publication June 7, 2004) 